\title{
LA CIENCIA ENTRE EL OBJETIVISMO Y EL CONSTRUCCIONISMO
}

\section{SCIENCE BETWEEN OBJECTIVISM AND CONSTRUCTIONISM}

Mg. Juan Carlos Aguirre (jcaguirre@unicauca.edu.co) Departamento de Filosofía. Universidad del Cauca (Colombia).

Dr. Luis Guillermo Jaramillo (ljaramillo@unicauca.edu.co) Facultad de Educación (Rudecolombia). Universidad del Cauca (Popayán, Colombia).

\section{Abstract}

This article proposes an alternative to the discussion between the objectivist and constructionist vision on science. In order to reach such objective, we start from a schematic presentation about what it is called Science Wars; then, we are going to talk in detail about Knor-Cetina's proposal, showing how it leads to antiscientific attitudes. Later, we will confront the constructionist thesis taken from Knor-Cetina with the realistic ones, proposed by Giere, Kitcher and Hacking, in order to moderate the antiscientific positions, cleansing the traditional polarities. Finally, the Critical Common-Sensism proposed by Susan Haack will be outlined and stated as an option to the confrontation between objectivists and constructionists and also, as a moderate way to understand science in the present-day.

Keywords: science, science wars, constructionism, critical realism, critical common-sensism.

\section{Resumen}

Este artículo propone una alternativa a la discusión entre las visiones objetivistas y construccionistas sobre la ciencia. Para alcanzar tal objetivo, partimos de una esquemática presentación de lo que se ha denominado las Guerras de la Ciencia; en seguida expondremos en detalle la propuesta de Knor-Cetina, mostrando cómo conduce a actitudes anticientíficas. Posteriormente, confrontaremos las tesis construccionistas extraídas de Knor-Cetina con las propuestas realistas de Giere, Kitcher y Hacking, con el fin de morigerar las posturas anticientíficas, depurando las tradicionales polaridades. Finalmente, esbozamos las tesis del Sensismo Común Crítico propuestas por Susan Haack, las que plantearemos como una salida a la confrontación entre objetivistas y construccionistas, así como una forma moderada de comprender la ciencia en la época actual.

Palabras clave: ciencia, guerras de las ciencias, construccionismo, realismo crítico, sensismo común crítico.

\section{Introducción}

La ciencia (1) es el fenómeno más impactante de la cultura moderna, pero a su vez, el más complejo; sus logros han deslumbrado generaciones, desafiado antiguos mitos y logrado lo que antes era sólo sueños de mentes visionarias. A través de la ciencia se ponen en juego las habilidades cognitivas más especializadas del 
ser humano; sus construcciones dan razón de un impresionante arsenal lógico y sus éxitos benefician amplios sectores de la sociedad. No obstante, la ciencia en tanto empresa humana, está expuesta al riesgo de la imprecisión, susceptible al razonamiento engañoso, motivada por el vaivén de los intereses personales o de los grupos de poder; en ocasiones, es considerada la causante de los peligros más radicales que amenazan el habitar del hombre y la mujer en el mundo, y al mismo mundo.

Ahora bien, el éxito de la ciencia permitió que la reflexión en torno a ella adoptara visos de optimismo, lo que dio origen a una apología y un deseo por entronizarla como saber auténtico, cuyo estilo y método debería seguir todo saber que quisiera considerarse válido y digno de ser transmitido y aprendido. A su vez, la prosperidad, rodeada de áulicos, ocultó posibles objeciones que hubieran enredado la consolidación del progreso científico. Sin embargo, con el trascurrir del tiempo, se fueron enunciando preguntas que ponían en jaque la imagen tradicional de la ciencia: ¿puede hablarse de método científico cuando lo que nos muestra la historia de la ciencia está lejos del ideal de rigor y orden?, ¿si el interés del científico es ir hallando verdades, cómo es que estas siempre están de lado del políticamente más fuerte?, ¿cómo decir que la ciencia usa lo más excelso del pensamiento cuando ni siquiera prevé la extinción del sujeto que piensa?, ¿acaso todo conocer puede englobarse en lo científico?, ¿dónde quedan las preguntas más urgentes de la especie humana?, ¿no es el científico simplemente un individuo entrenado para responder preguntas previas aprendidas de un manual?

Cuestionamientos como estos fueron tomando diversas formulaciones y alcanzando mayores espacios académicos en los más altos tonos; preguntas que se fueron refinando y dieron origen a tratados y grupos de investigación con igual o mayor peso que el que obtuvieron quienes se dedicaron a presentar una imagen de la ciencia en la era de la prosperidad y el progreso.

Este trabajo intenta dar cuenta de algunas tesis preponderantes tanto de quienes defienden a ultranza la ciencia -pues imaginan una ciencia motivada sólo por la lógica, la precisión, la verdad, el orden, lo inmutable-, como de quienes ven en ella el origen de todos los males que padece el hombre, un relato más, una narración que no tiene ninguna credencial más válida que la que tiene un cuento de Borges, la medicina ancestral o los oráculos de la Nueva Era. Los primeros corresponden a los objetivistas o cientificistas (2), los segundos a los construccionistas (3) que pregonan una manera "distinta" de hacer ciencia o anticiencia.

El objetivo no es elaborar un reducido estado del arte acerca de lo que se ha denominado en los últimos años las Guerras de la Ciencia (Science Wars), lo cual es difícil debido a los ríos de tinta que han corrido al respecto, aunque no por ello inútil; el propósito es mostrar la tensión vivida entre un amplio sector de las humanidades y las ciencias aplicadas. La intención no es ahondar en la radicalización de las posturas, sino en explorar una alternativa a la misma, esperando que esta permita abrir diálogos entre ambas concepciones de ciencia; diálogos que puedan contribuir teóricamente a ampliar horizontes de comprensión acerca de la Naturaleza de la Ciencia con todos los subtemas que ello implica; y, de modo práctico, en la aclaración del papel de las humanidades en los procesos de enseñanza de las ciencias.

Para llevar a cabo esta intención, esbozaremos algunas líneas generales que señalarán el terreno de debate que se ha desarrollado; posteriormente, mostraremos un caso concreto del ataque a la ciencia por parte de Karin Knor-Cetina, continuadora de las aproximaciones antropológicas y sociológicas a la ciencia, ataque que dirige al modo como los científicos llevan a cabo sus experimentos; seguidamente, plantearemos la respuesta de algunos reconocidos filósofos de la ciencia ante los desafíos de la sociología de la ciencia. De esta confrontación, se indicarán elementos que incrementan la polarización y algunas de las respuestas que 
se han generado. Finalmente, consideramos la propuesta que, gracias al agotamiento por las batallas libradas y la necesidad de una salida racional, puede emerger como alternativa para tiempos de tregua.

\section{Las Guerras de la Ciencia (Science Wars): unas breves indicaciones}

La expresión Guerras de la Ciencia ya ha entrado a formar parte de los clichés de los estudiosos de la ciencia. Su origen lo remontan algunos a finales de la década de los ochenta (ver Theocharis 1997) (4); sin embargo, la mayoría de los historiadores del fenómeno coinciden en que fue desde mediados de 1990 que la expresión alcanza su plena madurez (véanse los trabajos de Jardine y Frasca-Spada (1997) y Koertge (2007) que, separados una década, coinciden en que el detonante de estas guerras lo constituyó el libro de Gross y Levitt (1994). Dos fueron las piedras de toque de este debate:

La piedra de toque es el libro de Gross y Levitt, La más alta superstición: la izquierda académica y sus reclamos a la ciencia, planteaba con ojos críticos las pretensiones y los absurdos que se veían en trabajos recientes de los postmodernos norteamericanos, los (y las) feministas, los multiculturalistas y los ambientalistas radicales, especialmente en los campos de los estudios de la ciencia, es decir: historiadores, filósofos y sociólogos de la ciencia (Jardine y Frasca-Spada 1997:219); este libro generó una reacción en muchos ámbitos, destacándose - por su posterior papel en las Guerras de la Ciencia- el de la revista de estudios culturales Social Text, la cual dedicó un número completo a refutar las tesis de Gross y Levitt, aduciendo que ellos eran los apologetas del complejo militar e industrial que le habían declarado la guerra a los cultivadores de los Estudios de la Ciencia, los tecno-escépticos y los ambientalistas de Izquierda (Koertge 2007:105).

La segunda piedra de toque la constituye el famoso Affaire Sokal que en síntesis puede exponerse así: (a) dos físicos inquietos por la evolución intelectual que han experimentado ciertos medios académicos norteamericanos; (b) el "experimento no ortodoxo" de Alan Sokal al enviar a la revista Social Text, el artículo: Transgredir las fronteras: hacia una hermenéutica transformadora de la gravedad cuántica; (c) la aceptación y publicación del artículo en un número especial de esa revista dedicado a rebatir las críticas de varios científicos contra el postmodernismo y el constructivismo social; (d) la confesión posterior de Sokal que su experimento no ortodoxo era una broma, un artículo escrito plagado de absurdos, falto de lógica y enmarcado en el relativismo cognitivo extremo. Finalmente, (e) el escándalo en la prensa popular y en la comunidad académica (detalles de esto se ve en la introducción del libro de Sokal y Bricmont 1999). Años después del Affaire, el propio Sokal (1998) sopesa su alcance y saca algunas conclusiones:

(a) Sokal reconoce aspectos positivos de los estudios sociales de la ciencia, entre los que destaca: 1. La ciencia es una empresa humana y como cualquier empresa está sujeta a estricto análisis social; las cuestiones que a este respecto brotan son fértil campo de indagación para historiadores, sociólogos, politólogos, economistas. 2. El debate científico está restringido en parte por actitudes mentales prevalecientes, las cuales a su vez dependen de profundos factores históricos. 3. No hay nada malo con la investigación informada por un mandato político; de igual modo, tal mandato no ciega al investigador a la hora de determinar hechos inconvenientes (Sokal 1998:10).

(b) Sokal analiza bajo dos variables el alcance del artículo enviado a Social Text: la publicación de la parodia y el contenido del artículo. Respecto a la primera dice que su artículo no prueba que todos los estudios de la ciencia son meros sinsentidos, ni que los estándares de este tipo de publicaciones son generalmente laxos. En efecto, apoyados en esta experiencia, muchos críticos de los Estudios de la Ciencia niegan de plano el 
valor de cualquier investigación que en este campo se haga. A lo sumo, reconoce Sokal, el artículo prueba que los editores de la revista fueron negligentes al mandar a evaluarlo con pares expertos, tal vez suprimiendo este paso por el prestigio del investigador. Respecto a la segunda variable, es decir, el contenido del artículo, Sokal se reconoce orgulloso de él y muy divertido, aunque afirma que gran parte del contenido más hilarante lo otorgaron los maestros postmodernos.

(c) El contenido del artículo revela que gran parte de los maestros postmodernos utilizan un vocabulario trivial o no comprenden lo que leen: "primero que todo, se tienen enunciados absurdos o carentes de significado, palabras vacías, y la exposición de falsa erudición. Segundo, se tiene un pensamiento sentimental y una filosofía pobre que viene acompañada notablemente (aunque no siempre) en la forma de un relativismo superficial" (Sokal 1998:11). Ejemplos de sinsentido los encuentra Sokal en la asimilación de la teoría del caos que hace Deleuze y Guattari, la topología diferencial de Lacan e Irigaray, la cosmología de Lyotard, el tiempo no lineal en Serres; estos análisis los condensa en el libro Imposturas Intelectuales escrito en compañía de Bricmont. En el artículo Lo que prueba y no prueba el affaire Social Text, detalla los sinsentidos que se evidencian en la recepción que hace Latour de la teoría de la relatividad de Einstein.

(d) El pensamiento descuidado que critica Sokal manifiesta una confusión entre varios niveles, a saber: el ontológico (¿qué objetos existen en el mundo?, ¿qué enunciados acerca de estos objetos son verdaderos?), el epistemológico (¿cómo pueden los seres humanos obtener conocimiento de las verdades acerca del mundo?, ¿cómo se puede evaluar la confiabilidad de ese conocimiento?), la sociología del conocimiento (¿cómo están influenciadas las verdades conocidas (o cognoscibles) por los humanos, en cualquier sociedad dada, por factores sociales, económicos, políticos, culturales, ideológicos?), la ética individual (¿qué tipo de investigación debería hacer (o rehusar) un científico (o tecnólogo)?), la ética social (¿qué tipo de investigación debería cubrir, subsidiar o dar dineros públicos (o viceversa: no cubrir, cargar con impuestos o prohibir?) (Sokal 1998:14-15). Sokal reconoce que todos estos niveles están interrelacionados; sin embargo, entre ellos hay diferencias conceptuales que si no se tienen en cuenta, favorecerán el relativismo superficial.

(e) Sokal finaliza el balance considerando el construccionismo social y el relativismo presentes, especialmente, en el Programa Fuerte. Apoyado en una cita de Barnes y Bloor, en la cual se defiende y se explica el tipo de relativismo que adoptan. El autor comenta que ninguno de los sociólogos defiende un relativismo ontológico, sino que propugnan por uno de corte epistemológico: "que todas las creencias son igualmente creíbles, o igualmente racionales" (Sokal 1998:16); más aún, por alguna forma de relativismo metodológico para los sociólogos del conocimiento. La cuestión aquí es desentrañar ¿cuál es la forma de tal relativismo? Pudiera ser que se tratara de ciertos principios usados por la sociología y la psicología para explicar la causación de todas las creencias independientemente de nuestra evaluación en verdaderas o falsas, racionales o irracionales; en este caso, no ve Sokal ninguna objeción. El problema surge cuando se proclama que solamente las causas sociales pueden entrar a explicar las creencias y para nada cuenta el mundo (actitud que es predominante en la mayoría de las investigaciones de estos sociólogos y que lleva a explicaciones confusas o absurdas de lo que sucede en la ciencia).

Como conclusión, de este apartado, puede decirse que tanto Gross y Levitt como Sokal y Bricmont denunciaron al amplio público los abusos de los Estudios de la Ciencia, estudios que habían infiltrado la vida académica norteamericana. A su vez, hicieron que los críticos timoratos de estas posturas cada vez más enraizadas en la academia, hicieran un frente contra los excesos de los (utilicemos el mismo concepto que encerró las preocupaciones de Sokal y Bricmont) "postmodernos", encontrando un auditorio más 
concurrido. Continuando con la metáfora de la guerra (que para Sokal es errónea en tanto los editores de Social Text no son sus enemigos), los contendores en ella son: por un lado, los que consideran que la ciencia no es más que una narración, un mito, una construcción social; es decir, quienes representan una postura construccionista, que, obviamente, reaccionaron de modo airado contra Sokal y Bricmont (5); por el otro, los que ridiculizan tales posturas y defienden a ultranza la imagen científica tradicional. Aunque estos últimos dan la apariencia de moderación y racionalidad, caen también en excesos y simplificaciones, subvalorando preguntas hechas desde el flanco opuesto sin ofrecer respuestas satisfactorias.

\section{Una batalla librada en torno al laboratorio}

El recorrido hecho nos ha permitido distinguir los elementos esenciales de la discusión sobre la naturaleza de la ciencia y las razones de quienes se alzan como sus defensores, representados por Sokal. Veamos ahora, en este apartado, uno de los ataques de quienes estudian la ciencia desde los campos de la antropología y la sociología; crítica que se ocupa de la reflexión sobre el científico como experimentador, tema poco abordado por los filósofos de la ciencia anteriores a 1980 y terreno apropiado por los Estudios de la Ciencia actual, especialmente por los estudios construccionistas de la ciencia. El recurrir a este caso permitirá no sólo comprender parte de la reacción de Sokal, sino también, identificar los retos que estas corrientes han lanzado a la imagen tradicional de la ciencia; las respuestas a tales retos o bien han incendiado la discusión, o bien han permitido hallar puntos de encuentro para superar las visiones en conflicto.

El libro indicado para entablar el debate sería La vida en el laboratorio: la construcción social de los hechos científicos de Bruno Latour y Steve Woolgar (1979); sin embargo, no nos referiremos a este trabajo pionero sino a una versión posterior que utiliza la misma metodología propuesta por Latour y Woolgar, y llega a similares resultados, aunque quizás con mayor radicalidad. Haremos alusión exclusivamente a la socióloga Karin Knor-Cetina quien en 1981 escribió el libro La fabricación del conocimiento: un ensayo sobre el carácter constructivista y contextual de la ciencia. De este libro pretendemos resaltar sólo la pretensión epistemológica que da la autora a sus tesis, reduciendo al máximo cualquier referencia a la metodología que propone, una especie de etnografía de la ciencia (6), así como sus descripciones respecto a cómo se comportan los científicos en el laboratorio.

El punto central del ataque de los construccionistas lo constituye la debilitación de los hechos; para KnorCetina "los hechos no son algo que podamos dar por sentado, algo que pueda ser pensado como la roca sólida sobre la cual se construye el conocimiento. En realidad, su carácter es bastante problemático; tanto que, con frecuencia, si los confrontamos se esfuman" (p. 51). En este sentido, el construccionismo se opone al objetivismo para el que, en la definición de Knor-Cetina, "el mundo está compuesto de hechos, y el objetivo del conocimiento es proveer una versión literal de cómo es el mundo" (p. 51) (7). Obviamente, Knor-Cetina sostendrá que el objetivista olvida el carácter constituido de los productos científicos y que la facticidad no es más que una constitución del mundo a través del procedimiento de la lógica científica, sus explicaciones y validaciones. En este contexto, Knor-Cetina plantea que existen modelos de éxito que no necesariamente están apoyados en los presupuestos objetivistas, modelos no ajenos a las ciencias: "los psiquiatras, por ejemplo, han usado con mucha frecuencia terapias conductistas para tratar exitosamente desórdenes psiquiátricos mayores y menores de los que dicen no tener o necesitar ninguna explicación descriptivamente adecuada" (pp. 54-55). Las anteriores reflexiones llevan a Knor-Cetina a la conclusión que el presupuesto de un mundo factual estructurado a manera de leyes por conjunciones constantes de 
acontecimientos, no pasa de ser una suposición que sólo opera en el trabajo de laboratorio mas no en la práctica: "en la práctica, esas conjunciones constantes son las raras excepciones, raras como el éxito predictivo" (p. 55).

La conclusión a la que llegó Knor-Cetina nos plantea los siguientes cuestionamientos: ¿existe diferencia entre el mundo, llamémoslo, "real" y el mundo del laboratorio?, y si existe tal distinción: ¿acaso lo que se predice para el mundo del laboratorio opera en el mundo "real"? Hemos llegado a una situación problemática pues es casi obvio que el mundo real y el mundo del laboratorio son distintos: en el mundo del laboratorio hay asepsia, instrumentos, medidas exactas, condiciones libres de ciertas variables, etc. Ahora bien, si el mundo del laboratorio es, digámoslo, artificial, no tendríamos la posibilidad de correlacionarlo con el mundo "real" pues serían dos esferas distintas. En ese caso, tendríamos que concluir que el éxito predictivo en el laboratorio no sobrepasa el ámbito del laboratorio y que éste se convierte en el taller donde se fabrican hechos que no son los hechos del mundo sino el resultado de los experimentos del científico. De este modo, las leyes de la ciencia no son aproximaciones a la verdad, ni verosímiles, ni descriptivamente adecuadas, sino que son transfácticas y reglamentaristas. De este modo, lo que mueve a los científicos no es un razonamiento teórico sino un razonamiento práctico: “igual que con cualquier juego, el ganar depende menos de las reglas que de lo que se hace dentro del espacio creado por esas reglas" (p. 55).

El giro del razonamiento teórico al práctico abre a Knor-Cetina un campo de investigación, a su modo de ver, más prolífico en lo que tiene que ver con la formulación de una teoría empírica del conocimiento, pues se supera el mito del científico como descubridor de leyes de la naturaleza, como enunciador de lo que es, y se le ve inmerso en una trama de intereses, elecciones, transformaciones y construcciones que parten de lo que es. La confirmación de la distinción entre naturaleza y mundo del laboratorio, así como el desvelamiento del trabajo del científico reducido a juegos de intereses, llevan a Knor-Cetina a establecer sus premisas: (a) más que verdades epistemológicas, los científicos utilizan en el laboratorio funciones pragmáticas (8) y retóricas (9); (b) en la investigación se resalta el carácter político, que sobrepasa el posible credo del científico; (c) la teoría pura es una ilusión que la ciencia ha heredado de la filosofía.

Quizá, lo que más recalca este tipo de estudios (10), es lo denominado por Knor-Cetina la "carga de decisiones", o lo que otros podrían abordar desde la pregunta por la posibilidad de una "elección racional". Dice Knor-Cetina: “los procesos de fabricación [de los resultados científicos y los datos empíricos] involucran cadenas de decisiones y negociaciones mediante las cuales se generan sus resultados. Dicho de otra manera, necesitan que se haga una selección. Las selecciones, a su vez, sólo pueden hacerse sobre la base de previas selecciones: se basan en traducciones de otras selecciones" (p. 61). Exploremos este planteamiento: tenemos a un científico o grupo de investigación frente a un enigma (puzzle en sentido kuhniano); a su alrededor se encuentran variados instrumentos para responderla: literatura previa, conversaciones con colegas, herramientas de laboratorio, etc.; no existe un modo de respuesta; por tanto, deben elegirse los medios por los cuales se resolverá la pregunta y se enfrentará la investigación. En tanto no existe un camino trazado, el investigador deberá seleccionar sus medios y persuadir a la comunidad científica sobre sus resultados.

Las implicaciones que Knor-Cetina ve de este proceso selectivo es el campo que deja abierto a las alternativas, es decir, el científico eligió tal camino; sin embargo, los caminos se pueden desplegar en multitud de horizontes. Ahora bien: "si los objetos científicos son selectivamente extraídos de la realidad, se los puede deconstruir, cuestionando las selecciones que incorporan. Si los hechos científicos son fabricados, 
en el sentido de que son derivados de decisiones, pueden ser defabricados imponiendo decisiones alternativas" (p. 63). Trasladado al ámbito del laboratorio, tanto los hechos relevantes para la investigación como las formas de abordarlos responden a procesos de selección; si se supone que es el trabajo de laboratorio el que valida los enunciados, tal validación no conforma una opinión científica sino que constituye solidificaciones de hechos construidos en el laboratorio, exentos o debilitados de cualquier principio de racionalidad: "parece claro que no podemos esperar reducir estas situaciones a un número pequeño de criterios, y mucho menos a un principio de racionalidad que nos permitiría de allí en más predecir las selecciones del laboratorio del científico. Más bien, tendremos que tomar esas selecciones como producto de la co-ocurrencia e interacción de factores cuyo impacto y relevancia se constituyen en un tiempo y lugar dados, es decir, de las circunstancias en las cuales el científico actúa” (p. 70).

Podríamos decir que Knor-Cetina no observa con preocupación el hecho de que no existan criterios de selección y por tanto podríamos sucumbir ante el anything goes de Feyerabend. Para ella, la indeterminación no es ni extraña a la ciencia, ni perjudicial a ella; es más, sin tal indeterminación, el progreso de la ciencia sería impensable; gráficamente diferencia el proceso disruptivo en la ciencia con otras imágenes: el ruido en la teoría de la comunicación, los errores en el código genético, las perturbaciones en un sistema termodinámico; las disrupciones de las que habla Knor-Cetina son vistas más bien "como el sine qua non de una organización progresiva del sistema hacia una creciente complejidad, a pesar de los errores locales o las pérdidas de información" (p. 73).

Finalmente, esta aproximación al problema del laboratorio desde el punto de vista de la sociología construccionista de la ciencia, intenta, desde métodos que se pretenden científicos, explicar el por qué los científicos en el laboratorio no son razonadores teóricos, no buscan a toda costa aproximarse a la verdad, se esfuerzan por que sus experimentos tengan éxito y ellos prestigio, y otras muchas conclusiones poco exploradas por los filósofos obsesionados por las teorías (11). Las respuestas a este tipo de cuestionamientos han conformado un abanico que va desde posturas extremas que las califican de erróneas, pasando por aquellas que intentan aproximaciones desde una comprensión filosófica, hasta posturas que en el mismo terreno del experimento establecen acuerdos y desacuerdos. La presentación esquemática de cada una de estas posturas permitirá sugerir los modos posibles de salir de la dicotomía objetivismo-construccionismo.

\section{Filosofía de la ciencia y sociología de la ciencia: algunas salidas al falso dilema objetividad-construcción.}

En este apartado esbozaremos esquemáticamente tres respuestas dadas por algunos filósofos de la ciencia a los retos hechos por la sociología de la ciencia, retos ejemplarizados en el trabajo de Karin Knor-Cetina. La primera presenta algunas consideraciones de Ronald Giere (1994) en el artículo Viewing Science, en el cual se reduce al absurdo el presupuesto fundamental de la sociología construccionista de la ciencia. La segunda, considera el trabajo de Philip Kitcher (1998) A Plea for Science Studies; Kitcher modera las posturas antagónicas y propone salidas a la tensión que se da entre sociología y filosofía de la ciencia. Finalmente, se abordarán los artículos de lan Hacking (1988a y 1988b) Philosophers of Experiment y The Participant Irrealist at Large in the Laboratory en los que el diálogo se establece en el mismo terreno del experimento. Tanto Kitcher como Hacking serán puntales importantes para la posible alternativa a la tensión objetivismoconstruccionismo.

Para empezar, Giere (1994) hace una retrospectiva de las aproximaciones a la ciencia, algo así como un estado del arte que comienza con la década del veinte y culmina con los desarrollos de la década del 
ochenta. Los estudios de la ciencia los ubica en la década del sesenta (aunque reconoce a Merton como pionero de este tipo de investigación), década con grandes cambios sociales; por tanto, el antagonismo filosofía de la ciencia-sociología de la ciencia refleja para Giere "las diferentes experiencias de diferentes generaciones" (1994:8): la generación del treinta y la generación del sesenta. Respecto a la sociología construccionista de la ciencia, Giere reconoce la gran variedad de corrientes; no obstante, las sintetiza en dos tipos de construccionismo social: el construccionismo epistemológico y el ontológico. Para el primero, existe un abierto agnosticismo acerca de la existencia de entidades y procesos reportados por los científicos; para el segundo, las entidades y procesos nombrados en las discusiones científicas teóricas son constituidos por las prácticas sociales, las interacciones y las asociaciones de científicos (1994:8).

Aunque Giere reconoce que los estudios construccionistas son excitantes, sobre todo por la riqueza de sus casos mas no por sus aportes teóricos, identifica el problema nuclear en el principio metodológico de la reflexividad (12). La reflexividad es vista por Giere como una anomalía presente tanto para las versiones epistemológicas como para las ontológicas del construccionismo. Si se le preguntara a un construccionista social, p. ej. Karin Knor-Cetina, ¿cuál es el carácter de la sociología construccionista de la ciencia?, responderá que es una ciencia (recordemos que pretende encontrar explicaciones generales, ver nota 11). Si es una ciencia, la propia sociología de la ciencia tendrá que exponerse a los mismos tipos de investigación constructivista que se han aplicado a otras ciencias; en ese caso, "los constructivistas epistemológicos tendrían que concluir que sus propias creencias acerca de los científicos que ellos estudiaron, estaban determinadas más por sus propios intereses e interacciones sociales como sociólogos, que por lo que por sus propios sujetos investigados. Para los constructivistas ontológicos, los resultados de tales investigaciones podrían ser que los objetos de investigación, las creencias, intereses, etc., de sus científicos, son constituidos por sus propias prácticas como sociólogos constructivistas"' (Giere 1994:8).

Carecería de sentido, entonces, adelantar un estudio del tipo de Knor-Cetina, pues no dice nada del actuar de los científicos del laboratorio, sino de ciertas elecciones de un sociólogo y una manipulación de su fuente hasta hacerla decir algo que él quiere escuchar. Sin embargo, frente a la extinción del construccionismo a partir de su propia raíz, la reflexividad, algunos sociólogos prefieren celebrar la ironía reflexiva de la deconstrucción total, incluyendo la auto-deconstrucción (Wolgar 1988, citado por Giere 1994:8). Después de destruir el programa por sus propios postulados, Giere pasa a sostener que tal programa no es aceptable y a mostrar cómo la propuesta realista tiene prelación sobre él, pues es inmune a la enfermedad de la reflexividad: "en una visión realista, los científicos algunas veces tienen éxito al descubrir cómo es que realmente funciona cierta fracción del mundo, y [en los estudios de la ciencia anclados en la visión realista] podría tenerse éxito al explicar cómo es, de hecho, tal fracción del mundo" (Giere 1994:9). En tanto los estudios constructivistas de la ciencia tienen una anomalía de origen, éstos tendrían que adoptar una visión realista y dedicarse a explicar, desde este presupuesto, el éxito de la ciencia moderna (Giere 1994:10).

Por otra parte, Kitcher (1998) ofrece un tratamiento mesurado al problema de las guerras de la ciencia; su objetivo, tal y como lo reconoce al final de A Plea for Sciences Studies espera que se puedan trascender las guerras de la cultura y el debate sirva para que existan enfoques más productivos a los importantes problemas que están en juego. La exposición de Kitcher en ese texto puede sintetizarse en tres bloques: el primero, los alcances no teóricos sino reales que han alcanzado los estudios de la ciencia; el segundo, los grupos de presupuestos que deben adoptarse, sin glosas, en la discusión; finalmente, las causas de los excesos de los estudios de la ciencia. Para esta exposición invertiremos la presentación de tales temas. 
Textos como el de Knor-Cetina conllevan a un relativismo que, a decir de Kitcher, es un camino pavimentado "con las mejores intenciones pero con los peores argumentos" (1998:44). Los presupuestos asumidos como dogmas por los sociólogos construccionistas de la ciencia fueron tomados acríticamente de las reflexiones de la filosofía de la ciencia. El primer dogma responde a la carga teórica de la observación, este dogma se transforma en sociología de la ciencia como que no hay verdad excepto la aceptación social: "la tentación es pretender que encontramos en la naturaleza sólo lo que pongamos en ella; que el mundo -o al menos el único mundo del que podemos hablar con sentido- es formado o construido por nosotros" (Kitcher 1998:38). El segundo dogma se forja a partir de la indeterminación (underdetermination) de la teoría por la evidencia; su formulación en la sociología de la ciencia es: no hay sistema de creencias que esté restringido por la razón o la realidad, y ningún sistema de creencias es privilegiado. El tercer dogma argumenta a partir de la tesis de la variedad de la creencia: dirán los sociólogos de la ciencia: "supongamos que partimos del hecho evidente que las personas, incluyendo los científicos, algunas veces difieren en sus creencias. ¿Cómo podemos explicar este hecho? No puede ser apelando al mundo, pues la naturaleza que los creyentes confrontan es la misma en ambas instancias" (Kitcher 1998:41). Este planteamiento apoya la pretendida asimetría del Programa Fuerte, es decir, no debería haber asimetrías en la explicación de la verdad o la falsedad, la sociedad o la naturaleza. Finalmente, el cuarto dogma surge al considerar la "categoría actor" y la escritura de la historia: "al contar una historia de un desarrollo científico, no debemos emplear conceptos que no estén disponibles a las personas involucradas" (Kitcher 1998:43); esto implica que el honor siempre debe darse a las categorías de los actores.

Ahora bien, señalados los errores que invaden la mayoría de los estudios de la ciencia, es preciso superar el ámbito de las mutuas recriminaciones y poder encontrar puntos básicos de discusión a partir de los cuales construir fecundos debates. Kitcher agrupa estos presupuestos (que él llama incontrovertibles) en dos: 1) el grupo realista-racionalista, en el cual se presupone que (a) la ciencia es progresiva y este carácter progresivo se manifiesta en poderes crecientes de predicción e intervención; (b) la predicción e intervención permiten que se postule que las clases de entidades descritas en la investigación científica existen independientemente de nuestro teorizar y que muchas de nuestras descripciones son aproximadamente correctas; (c) nuestras pretensiones son vulnerables a futuras refutaciones; (d) en las áreas más prominentes de la ciencia, las discusiones descansan en la evidencia y en las disputas se apela a cánones de razón y evidencia; (e) los cánones de razón y evidencia también progresan con el tiempo (Kitcher 1998:3436). 2) El grupo socio-histórico que comprende: (a) la ciencia es hecha por seres humanos; (b) ningún científico llega al laboratorio libre de categorías y preconceptos; (c) las estructuras sociales presentes en la ciencia afectan los modos en los que la ciencia es recibida y transmitida; (d) las estructuras sociales en las que la ciencia se desenvuelve afectan los tipos de preguntas que se toman como las más significativas, así como, algunas veces, las respuestas que son propuestas y aceptadas (Kitcher 1998:36-38). Del equilibrio de estos dos grupos depende que pueda establecerse un diálogo fructífero.

Lo esbozado del planteamiento de Kitcher partió de la identificación de unos precarios fundamentos de los estudios de la ciencia y continuó con unos presupuestos que recogían los elementos básicos de la discusión esgrimidos por cada uno de sus contendores. El objetivo ahora consiste en integrar ambos grupos sin radicalizar uno en desmedro del otro; en este caso, el falso dilema entre objetivismo y construccionismo, se superaría al entablar diálogos en los que se conserven los puntos incontrovertibles, reconociendo los excesos que puede comportar cada postura, sin que ello implique caer en el extremo que se confronta, ni en una posición complaciente para ambas. Precisamente, Kitcher defiende un debate que supere el contexto de las guerras de la ciencia, lo que exige reconocer, entre otras cosas, que los ataques dirigidos a los 
estudios de la ciencia han consistido en diatribas y generalizaciones que impiden reconocer las posibilidades de estos programas (13), así como los logros obtenidos por estos investigadores (14).

La última postura a considerar es la del filósofo de la ciencia lan Hacking, quizá uno de los pioneros en la reflexión sobre la naturaleza experimental de la ciencia. A diferencia de los filósofos anteriores, Hacking responde a los sociólogos construccionistas de la ciencia en su propio terreno, el cual involucra como tema central el laboratorio. Hacking (1988a) plantea que la filosofía de la ciencia, hasta mediados de los ochenta, no había desarrollado suficiente literatura con relación a la filosofía del experimento; sin embargo, a partir de esa época tal reflexión ha ido en constante avance. La filosofía del experimento complementa la reflexión de la filosofía de la ciencia la cual, a decir de Hacking, "ha estado empobrecida no sólo por su obsesión con la teoría, sino también con su doctrina complaciente de que hay uno o al menos dos tipos de teorías (las reales y los principios conectores)" (1988a:148). La reflexión sobre lo experimental mejorará la calidad de vida de las teorías.

Con relación al tema que nos ocupa, Hacking distingue dos tipos de actitudes de los científicos ante el experimento: los escépticos y los admiradores. Entre los primeros están los sociólogos del Programa Fuerte en la sociología de la ciencia, aunque el alcance de estos es menor comparado con el movimiento denominado escuela de la "construcción social de los hechos científicos", inaugurada por Latour, de la cual, Knor-Cetina es una alumna eximia. El éxito del análisis de Latour consistió en que el líder del grupo de investigadores que trabajaban en el laboratorio investigado etnometodológicamente por Latour alcanzó, en 1977, el premio Nobel en medicina; así que La vida en el laboratorio se convirtió en una especie de diario de campo acerca de un científico de punta, ganador del Nobel.

El libro de Latour y Woolgar fue leído por grandes mayorías de un modo antirrealista: "la investigación [llevada a cabo por los científicos del laboratorio de Texas] alcanzó una conclusión exitosa no porque los investigadores que estaban en competencia produjeron una sólida evidencia, sino porque negociaron con la gran comunidad de endocrinólogos y forzaron la aceptación de sus análisis y síntesis de un tripéptido particular" (Hacking 1988a:151). El alegato de Latour y Woolgar no se centra tanto en adoptar una postura antirrealista acerca de las entidades inobservables, cuanto en sostener que ellas no existen hasta que sean construidas (o fabricadas, para conservar el término de Knor-Cetina). Obviamente, Hacking está alineado entre los admiradores de la ciencia y, por ende, el entusiasmo que tuvieron los lectores iniciales de La vida en el laboratorio, no causó ningún efecto en él. Sin embargo, esta falta de entusiasmo no lo privó de un análisis serio de la obra de los sociólogos construccionistas de la ciencia (15); al contrario, lo retó para leer el libro de modo atento y crítico, concluyendo que la historia "construccionista" puede volver a enunciarse de un modo completamente no-construccionista (de modo detallado puede verse esta traducción del antirrealismo al realismo en Hacking 1988b). Más que los argumentos que permitieron a Hacking mostrar cómo lo visto por Latour, Woolgar, Knor-Cetina y otros, puede ser leído desde la perspectiva del realismo, nos interesa ver cuál fue su actitud al respecto, a sabiendas que su interés era más moderado que descalificador de los estudios de la ciencia.

En primer lugar, Hacking desafía las viejas concepciones de verdad asumidas por los que podrían llamarse, a la luz de este trabajo, objetivistas: "la descripción, dice el pensador conservador, siempre era la verdad del mundo, y no una verdad fabricada"; frente a esto Hacking afirma que este pensamiento excluye otro tipo de descripciones, a pesar de que no hay una descripción correcta, sino que ésta es sólo un satisfactorio metahecho acerca del mundo y sus descriptores (16). El pensar así no comporta necesariamente que uno se 
tenga que apoyar en el subjetivismo. En segundo lugar, la lectura que hace Hacking acerca del experimento concede ciertos elementos, sin caer en el extremo construccionista. Según Hacking, es cierto que existe una creación del fenómeno en el laboratorio (17), que el fenómeno se purifica y mantiene en el laboratorio en unas condiciones que no existen en ninguna parte del universo; sin embargo, "esta noción de purificar, crear y regularizar el fenómeno [...] involucra ciertamente pensar y teorizar acerca del mundo material; pero esto también involucra interacción con el mundo, y, en un sentido no metafórico de la palabra, rehacerlo" (Hacking 1988a:152). Ahora bien, ni el mundo le fija al científico cuáles descubrimientos o invenciones puede hacer, ni el conocimiento pasado establece lo que cuenta como descubrimiento; este mundo no está pre-ordenado ni lógica ni históricamente y la ciencia puede abordarlo desde distintos caminos, creando diferentes fenómenos; esto implica que el camino responde a un momento específico en la historia y, por tanto, en otro camino de la historia podría ser de otro modo.

Finalmente, Hacking critica la imagen de ciencia dada por la filosofía orientada hacia la teoría, la cual aparecía no sólo como totalidad hegemónica unificada, sino también como inerte y pasiva. La unidad de la ciencia en la actualidad se articula alrededor de técnicas estadísticas endémicas, las computadoras rápidas que transforman la articulación de la teoría, el procesamiento de datos y la simulación de lo sintético; sin embargo, existe un gran unificador: los instrumentos y los aparatos. A diferencia de lo que consideran los sociólogos construccionistas de la ciencia, los aparatos no se traslapan de disciplina a disciplina porque son costosos o porque uno es más económico que otro (18); no se escogen con el fin por azar o capricho: "Ios espectrómetros nucleares de resonancia magnética, que estuvieron una vez en las fronteras de la física nuclear, son ahora los fundamentos de los ensayos bioquímicos. No porque el bioquímico piense que la física usada para hacer el aparato sea correcta, sino porque los instrumentos están allí, los únicos para usarse" (Hacking 1988a:153).

En conclusión, tanto Giere como Kitcher y Hacking han confrontado los planteamientos nucleares de los estudios de la ciencia; el abordaje hecho por cada uno va desde el desnudamiento de sus falencias, pasando por el reconocimiento de las condiciones para un diálogo fructífero y la enunciación de los logros de un programa para muchos relativista e irracionalista; finalmente, terminamos con una actitud abierta a las nuevas posibilidades que se le abren a la reflexión (filosófica) sobre la ciencia, aunque crítica con relación a la vieja imagen de ciencia centrada en la teoría, así como a los construccionistas que enfatizan un lado de la historia. El esquemático recorrido por estas tesis permitirá, anclados en ellas, sugerir algunos elementos que permitan adoptar una posición lejana tanto al objetivismo como al construccionismo.

\section{Alternativas al falso dilema}

En el transcurso de la discusión se ha observado cómo las posturas han variado de tono: de un aparente estadio de inconmensurabilidad, a una lectura más moderada por parte de los defensores de la ciencia; sin embargo, un estado del arte actual nos llevaría a sospechar que esta variación de posturas ha ocurrido más en algunas huestes de la filosofía de la ciencia que en otras disciplinas humanistas: antropología, sociología, historia de la ciencia e, inclusive, ciertos sectores de la filosofía de la ciencia. Aún hoy puede notarse, más en nuestro contexto, cómo desde las humanidades se ataca la ciencia, viendo en ella un peligro que sólo podría ser solucionado por las mismas humanidades. Para sobredimensionar el carácter de peligroso, algunas disciplinas hacen una lectura caricaturesca de la ciencia; caricaturas que quizás alguna vez pudieron haberse formado al interior de los defensores de la ciencia, pero que ellos mismos se encargaron de impugnar. En tal sentido, las humanidades en la ciencia no cumplirían otro rol que el de servir de trinchera a quienes 
reciamente la atacan (19). A continuación presentamos algunas consideraciones que podrían permitir una relación más provechosa entre las humanidades y las ciencias básicas y aplicadas, lo que permitiría comprender que necesariamente no se tiene que estar en una de las dos orillas.

1. Explorar la naturaleza de la ciencia haciendo que en la reflexión confluyan diversos factores de modo integrado. Después de años de debate, la naturaleza de la ciencia no puede ser la misma que se defendía en el período entreguerras (20), aunque tampoco es la que intentó presentar Lyotard (1989) (21). A este respecto Kitcher (2001) hace el siguiente diagnóstico: “Hace un tiempo, en aquellos queridos días lejanos, existía una concepción de la ciencia hoy casi (aunque no totalmente) enterrada en el olvido, y que contaba con aprobación generalizada tanto popular como académica. Tal concepción merece un nombre. La llamaré 'Leyenda'. La Leyenda ensalzaba la ciencia. Al describir a las ciencias como enfocadas a alcanzar objetivos nobles, sostenía que esos objetivos se han ido alcanzando cada vez con mayor éxito. Para explicar esos éxitos no tenemos que buscar más allá, nos dice, de las ejemplares cualidades intelectuales y morales de los héroes de la Leyenda, los grandes artífices de los grandes avances. La Leyenda ensalzaba tanto a los científicos como a la ciencia" (2001:14).

El diagnóstico de Kitcher, sin embargo, pese a explorar la naturaleza de la ciencia (específicamente en sus características de racionalidad y progreso) y a deshechizarla de sus caracteres legendarios, no sucumbe a las tentaciones irracionalistas, relativistas o subjetivistas. Unas humanidades comprometidas codo a codo con la praxis y la teoría de la ciencia permitirá una mejor comprensión de su naturaleza, cuestión no sólo importante para los deleites solipsistas del humanista, sino útil para la motivación de nuevas generaciones en la enseñanza de las ciencias.

2. La exploración de la naturaleza de la ciencia conlleva una desacralización de lo que se ha denominado método científico. Quizás con relación a esto puedan servir los planteamientos de la epistemóloga Susan Haack (2007) quien, precisamente para resolver el dilema entre lo que ella llama viejo diferencialismo y nuevo cinismo, sostiene: "La investigación en las ciencias es continua con otras clases de investigación empírica. Pero los científicos han ideado muchas y variadas formas de incrementar y refinar las fuentes de las que depende toda nuestra investigación empírica cotidiana. Los experimentos controlados, por ejemplo -algunas veces pensados como el distintivo de las ciencias- no son usados por todos los científicos, o sólo por los científicos; los astrónomos y los teóricos de la evolución no los usan, pero los mecánicos de autos, los plomeros y los cocineros sí lo hacen. En muchas áreas de la ciencia, sin embargo, las técnicas de control experimental han sido desarrolladas con un refinado arte (2007:95) (22).

La diferencia, entonces, entre los métodos de la ciencia y los métodos usados en la cotidianidad reside en que aquéllos involucran un refinamiento del pensamiento cotidiano. Esto nos lleva a la última consideración que serviría de alternativa crucial a la solución de lo que hemos denominado: falso dilema.

3. Es curioso advertir que existe una recurrencia de ambos bandos en discusión por apelar al sentido común; prácticamente cientificistas y atacantes (voluntarios e involuntarios) de la ciencia intentan persuadir al lector de que sus conclusiones deben ser adoptadas pues están basadas no en razonamientos forzados, sino en el mismo razonamiento del sentido común, es decir, en el razonamiento que cualquier individuo puede hacer sin mayor entrenamiento en las artes de la lógica (ver al respecto Aguirre y Jaramillo 2009) (23). Sin embargo, pese a que la ciencia parte del sentido común, es imposible desligar de ella su connotación crítica (24). Quizás por ello sea importante agregar a la recurrida expresión sentido común, la palabra crítico (25). 
Las características de un sentido común crítico serían:

(a) Reconoce que la investigación genuina, a diferencia de la investigación entorpecida por los intereses (políticos, económicos, culturales, de género), aunque no exenta de ellos, es un esfuerzo bien-intencionado por llegar a la verdad en cuestión, no importando el color que esa verdad pueda tener.

(b) Distingue entre investigación mejor y peor conducida: la primera se diferencia de la segunda pues es más crítica, más completa, más imaginativa, más creativa.

(c) Admite que aunque se busque bien-intencionadamente la verdad, la evidencia es siempre incompleta y ramificada; a menudo potencialmente desencaminada, y frecuentemente ambigua. En la investigación no hay garantía de éxito y el rigor del trabajo conlleva la tentación de ahorrar esfuerzos con base en la presión de quienes la financian.

(d) Acepta que no hay una lógica estándar para conducir una investigación. Los investigadores "hacen conjeturas informadas acerca de la explicación posible del fenómeno que les preocupa, revisan cuán bien estas conjeturas se compaginan con la evidencia que ya tienen y con la evidencia posterior que pueden acopiar, y luego usan su juicio bien sea para conservar su conjetura, derribarla o modificarla. Ellos necesitan imaginación, pensar en explicaciones posibles a los fenómenos problemáticos, concebir maneras de adquirir la evidencia que necesitan e imaginar fuentes potenciales de error; cuidado, habilidad y persistencia; buscar evidencia relevante que nadie tiene aún, así como evidencia relevante que otros tienen; honestidad intelectual, la fibra moral para resistir la tentación de salirse del camino de la evidencia que podría debilitar sus conjeturas, o manipular evidencia desfavorable que no se puede evitar; razonamiento riguroso, imaginar las consecuencias de sus conjeturas; y buen juicio al evaluar el peso de la evidencia, liberado de deseos, o miedos, o esperanzas de resolver rápidamente un caso o de agradar a un patrón o mentor o de convertirse en rico y famoso" (Haack 2007:97).

(e) Lo anterior no puede ser posible sino a través de modelos creativos que permitan al investigador moverse críticamente en un problema determinado y no ser obturado por modelos de relación lineal-causal o circular (26). Ello no quiere decir que los modelos existentes no sean importantes para ayudar a resolver un problema; sin embargo, a nuestro juicio, un modelo no se aplica fiel copia a un problema determinado en tanto las realidades son disímiles y las percepciones y experiencias que se viven en el trabajo de campo son de manera singular. En este sentido, lo importante es cómo los investigadores conocen las explicaciones epistemológicas de los modelos y métodos de sus proyectos, así como su justificación en la solución o resolución del problema. De esta manera, ellos se colocan en situación y con intencionalidad respecto a qué tipo de modelos y métodos son los más pertinentes y que cambios heurísticos habrán de efectuarse en sus procesos de investigación (Jaramillo 2006).

(f) Finalmente, el sentido común crítico conlleva la idea que los investigadores utilizan ayudas científicas para la investigación, sin que esto implique, tal y como podría deducirse de la lectura del trabajo de KnorCetina, que el carácter local y progresivo de las ayudas científicas (instrumentos de laboratorio) revela que los estándares de investigación bien conducida son relativos al contexto o paradigma. Al contrario: "toda investigación empírica depende de la experiencia; pero entre los modos en los que la investigación en las ciencias es a menudo 'mejor' está el que la experiencia de la que depende no está sin asistencia sino mediada por instrumentos de todo tipo" (Haack 2007:101). Los instrumentos, sin embargo no figurarán la realidad o la crearán, como sostienen los construccionistas; ni tampoco se convierten en moles cerradas a la 
crítica; ellos están abiertos al escrutinio por otros investigadores, es decir, no se deja el análisis de los instrumentos al azar o a la serendipia, sino que responde a criterios deliberativos, ingeniosos y controlados.

Las discusiones tejidas en nuestro contexto alrededor de la ciencia poco a poco han ido tomando las connotaciones que dieron origen a las guerras de la ciencia: van surgiendo recientemente posturas anticientíficas camufladas en discursos de multiculturalidad, decolonialidad, postcolonialidad; no ha faltado, tampoco quien se levante a denunciar los excesos de irracionalidad de infiltraciones postmodernas en la academia y el fatídico nicho que ellas tienen en las humanidades. No obstante, más que repetir la disputa que ocupó casi tres lustros, sería más provechoso recoger las enseñanzas del debate entre objetivistas y atacantes de la ciencia y proponer visiones alternativas a tal dicotomía.

\section{Notas}

(1) El presente artículo hace parte de las discusiones del grupo de investigación: Fenomenología y Ciencias; reflexiones que se vienen desarrollando desde el año 2004 y que se consolidan en líneas teóricas que involucran tanto cuestiones epistemológicas, como rutas de acción en Investigación para las Ciencias Sociales y las Didácticas de las Ciencias.

(2) Adoptamos sin glosas la definición dada por el DRAE de Cientificismo, a saber: "1. Doctrina según la cual los métodos científicos deben extenderse a todos los dominios de la vida intelectual y moral sin excepción. II 2. Teoría según la cual los únicos conocimientos válidos son los que se adquieren mediante las ciencias positivas. || 3. Confianza plena en los principios y resultados de la investigación científica, y práctica rigurosa de sus métodos. I| 4. Tendencia a dar excesivo valor a las nociones científicas o pretendidamente científicas".

(3) Hemos intentado presentar aquello que pregona la construcción social de la ciencia, bajo el concepto "construccionista", intentando desligarlo del "constructivismo", más cercano a ciertas escuelas psicológicas. Sin embargo, dado que los autores abordados utilizan en ocasiones la palabra constructivism y constructionism de manera indiferenciada, se respeta el uso original del autor abordado.

(4) En esta carta Theocharis refuta el nacimiento de las guerras de la ciencia a mediados de la década del noventa, arguyendo que en 1987 en la revista Nature, del 22 de mayo, aparece la siguiente mención: “Desde hace dos años, la comunidad académica norteamericana se encuentra encallada en un acalorado debate acerca de los fundamentos filosóficos de la ciencia. La publicación de esta semana da cuenta de las tendencias de lo que se ha conocido como 'las guerras de la ciencia' y describe cómo en el Reino Unido han surgido problemas similares" (Theocharis, 1997:271). Termina Theocharis su carta sosteniendo que de haberse atacado el fenómeno de la anticiencia en las décadas del setenta y ochenta, las guerras de la ciencia hace mucho se hubieran ganado, sin tanto daño a la academia y a la sociedad.

(5) Varios títulos mostrarían lo candente del debate hacia finales de la década del noventa; baste citar algunos: la compilación hecha por Baudoin Jurdant (2003) bajo el título: Imposturas científicas; los malentendidos del caso Sokal. Las propias defensas de Derrida tituladas: Sokal y Bricmont no son serios y Descomposturas Intelectuales; estos y muchos títulos más pueden encontrarse en la propia página de Sokal: http://www.physics.nyu.edu/faculty/sokal/, así como en la página oficial de las guerras de la ciencia: http://sciencewars.tripod.com/ 
(6) La metodología propuesta por Knor-Cetina puede resumirse en (a) la propuesta de una "metodología sensitiva" (MS) en oposición a lo que denomina "metodología fría"; (b) La MS rechaza la distancia e indiferencia del investigador, su desinterés con los fenómenos estudiados y la neutralidad; en la MS prima la intersubjetividad metodológica; (c) a la MS no sólo le basta con comprender el fenómeno, sino también con controlar la constitución conceptual dada a esa comprensión en la representación o en la transmisión de los constreñimientos (ella denomina este proceso "dejar hablar"). Finalmente, (d) la MS defiende un interaccionismo metodológico (2005:87-109).

(7) En este punto Knor-Cetina parafrasea una cita de van Fraassen (1977) para definir al realista científico; esta definición posteriormente aparecerá en su conocida obra The Scientific Image (1980): “la Ciencia pretende darnos, en sus teorías, una historia literalmente verdadera de cómo es el mundo; la aceptación de una teoría científica involucra la creencia que es verdadera" (van Fraassen 1980:12). Es necesario hacer dos apreciaciones: aunque Knor-Cetina se apoya en van Fraassen, no quiere decir que el Empirismo Constructivo de van Fraassen (antirrealismo instrumentalista) tenga que ver con el antirrealismo científico de los construccionistas (esta aclaración puede verse en Hacking 1988a:151); además, la definición que KnorCetina da del objetivismo, así como la definición que da van Fraassen de realismo científico, pueden considerarse precarias para entender la complejidad del tema. Una crítica a este respecto se encuentra en Giere (2005).

(8) En este punto afirma: "Si hay un principio que parece gobernar la acción del laboratorio es la preocupación de los científicos por que las cosas 'funcionen', lo cual apunta a un principio de éxito, más que de verdad" (Knor-Cetina 2005:59).

(9) Knor-Cetina sostiene: “Lograr hacer que las cosas 'funcionen' es una búsqueda mucho más mundana que la de la verdad, y una búsqueda en la cual la vida cotidiana de la ciencia es constantemente convertida en créditos mediante la publicación" (Knor-Cetina 2005:55).

(10) Nos referimos aquí no solo a los Estudios de la Ciencia (Science studies), sino también a las Sociologías y Antropologías constructivistas de la ciencia e, incluso, al Programa Fuerte (Strong Program) en Sociología de la Ciencia.

(11) Las críticas a esta obsesión por la teoría, tan típica de la mayor parte de la filosofía de la ciencia, pueden encontrarse en Hacking (1983 y 1988a).

(12) Según los sociólogos de la ciencia pertenecientes al programa fuerte, existen cuatro principios metodológicos a la hora de adelantar los estudios de la ciencia: causalidad, imparcialidad, simetría y reflexividad. Este último presupuesto, el más problemático según Giere, es definido por un miembro fundador del programa fuerte así: "En principio, sus patrones de explicación deberían ser aplicables a la sociología misma. Como el requisito de simetría, ésta es una respuesta a la necesidad de buscar explicaciones generales. Se trata de un requerimiento obvio de principio porque, de otro modo, la sociología sería una refutación viva de sus propias teorías" (Bloor 1998:38).

(13) Dentro de las posibilidades de las perspectivas históricas, filosóficas y sociológicas Kitcher (1994) resalta: los análisis valiosos de cómo emergió la comprensión científica contemporánea, la clarificación metodológica y conceptual, especialmente, en las áreas de disputa teórica; la conciencia de las presiones 
sociales que afectan cierto tipo de investigación científica; los aportes a una política científica más racional gracias a las investigaciones sobre el impacto de los hallazgos científicos sobre los individuos y la sociedad.

(14) De los cuatro logros reseñados por Kitcher (1994) resaltamos dos, que podrían estar en consonancia con el trabajo de Knor-Cetina: (a) el trabajo filosófico sobre el debate sociológico, la controversia sobre el IQ, la controversia sobre las unidades de selección, las implicaciones del teorema de Bell y la metodología causal de las ciencias sociales; (b) la investigación socio-histórica sobre los modos en los que excluir ciertas clases de personas de la investigación científica se ha afectado el carácter de la ciencia que se hace.

(15) En Hacking (1988b) puede verse su preocupación por las afirmaciones contenidas en estos estudios. Para él, La vida en el laboratorio es "quizás el más poderoso tratado antirrealista". El propio Hacking siente culpa al no abordar en su clásico libro Representar e Intervenir este reto y reconoce que 1988b es un intento por enmendar ese error. Es importante resaltar que en este artículo Hacking enfatiza más en las características del libro que a él le parecen más poderosa y deja de lado aquellas que podrían parecer débiles; a diferencia de muchos lectores de ese texto, Hacking sostiene: "quiero que los filósofos de la ciencia tomen este libro muy seriamente, y no (si son anti-construccionalistas) lo desechen junto con el dogma o la política que detestan" (1988b:278).

(16) En este punto Hacking formula lo que llama teoría realista de la verdad por correspondencia, la cual "debería suponer que el universo es mucho más complejo para que las personas describan todas sus partes o para producir una teoría unificada de él. En vez de eso podemos imaginar una colección inagotable de descripciones del mundo las cuales son independientes unas de otras. Cada descripción es verdad del mundo y [...] corresponde a algunos aspectos de la estructura del mundo" (1988b:291).

(17) Para ilustrar la afirmación que hay "creación de fenómenos" Hacking recurre a un caso de la inmunología: los investigadores han implantado satisfactoriamente grandes porciones del sistema inmune humano en ratones, dándoles un pequeño modelo funcional que puede ser usado para comprobar drogas y vacunas $y$, sobre todo, para tratar de investigar el sistema inmune humano, sin necesidad de hacer experimentos con humanos. Se partió de un hecho: la carencia de un sistema inmune por parte de los ratones; sin embargo, estos sobreviven gracias a la intervención de los experimentos: "este fenómeno, del sistema inmune humano viviendo en unos ratones defectuosos genéticamente es nuevo en el universo" (Hacking 1988a:152). De igual modo, Hacking sostiene que son los filósofos que no saben nada acerca del experimento los que dudan acerca de la creación del fenómeno (1988b:285).

(18) Knor-Cetina es divertida al consignar las respuestas dadas por los científicos ante la pregunta: ¿por qué se eligió tal instrumento para tal propósito? Las respuestas fueron variadas: "porque es caro y raro y quiero conocerlo" o "es más económico en términos de energía", o "John lo sugirió y me mostró cómo usarlo", o "estaba por ahí, así que era lo más fácil" (Knor-Cetina 2005:70).

(19) Desde hace un par de décadas el filósofo norteamericano John Searle ha señalado la crisis que los discursos de los estudios culturales han causado en la educación superior norteamericana; para este análisis ver Searle (1993).

(20) Una de las críticas a esta imagen de ciencia fue expuesta por Hacking (1988a): "1905 ha sido llamado el annus mirabilis, el año en el que Einstein expuso no sólo la teoría especial de la relatividad, sino también la explicación del fotón, y la plena comprensión del movimiento Browniano en la mecánica estadística. En 
noviembre de ese año el Rey de Portugal, un mecenas de la ciencia y la literatura, hizo una visita a París. Allí se le presentó una estatua de Brassais, de un metro de altura: nature se decouvrent devant la science. Una joven mujer, la ciencia, está cubriendo su cabeza con una capa, desvistiéndose a sí misma para revelar su pecho expuesto y su delgado cuerpo" (p. 153).

(21) Algunas de las tesis de Lyotard (1989) son las siguientes: "El que la ciencia sea un relato regido por unas reglas de juego convenidas por los científicos, hace que sea un relato entre otros que tienen las mismas características. La ciencia, el arte, la religión se entienden tradicionalmente como narraciones que contienen el saber: 'el relato es la forma por excelencia de ese saber'; por tanto, el saber no es exclusivo del relato científico pues está también contenido en los demás juegos de lenguaje que conforman la cultura. Más que prelación de la ciencia, a lo que asistimos hoy es a una pluralidad de juegos de lenguaje. Lyotard establece la diferencia que se ha marcado por siglos (desde la Revolución Copernicana) entre saber científico y saber narrativo; según él, el saber científico exige el aislamiento de un juego de lenguaje, excluyendo a los demás; al aislarse, la ciencia deja de hacer parte de la trama social, pasa a ser un mero componente indirecto; los enunciados del saber científico se justifican no tanto por lo que se enuncia, sino por el puesto del enunciador y las estrategias que usen para darles apariencia de verdad; la ciencia aspira a un progreso y, por ello, su único lema es el proyecto que marca el ritmo y el acople con la naturaleza" (Aguirre 2007:4 ). Para una exposición más amplia de las ideas de Lyotard, así como de las dificultades que entrañan ver Aguirre (2007).

(22) Pareciera un tanto débil esta posición con respecto al método científico; sin embargo es importante destacar que Sokal, el físico que se alzó como el defensor por excelencia de la ciencia, recoge en 2007 la misma idea de Haack. Sokal sostiene: "ciencia (tal y como yo uso el término) es practicada rutinariamente no sólo por los físicos, químicos y biólogos, sino también por los historiadores, los detectives, los plomeros e, incluso, por todos los seres humanos en (algunos aspectos de) nuestra vida cotidiana" (2007:3).

(23) Un intento de aclaración de términos en torno a la relación entre ciencia y sentido común puede encontrarse en Aguirre y Jaramillo (2009). Se muestra allí cómo una crítica constante a la ciencia nacida en la modernidad consistió en su separación del mundo de la vida; sin embargo, la lectura de este concepto dio pie para propuestas como la de Maffesoli (1993 y 1997) que caían en un sentido común, digamos, ingenuo. El apelativo de ingenuo se da a partir de Popper (1992).

(24) En Jaramillo y Aguirre (2004) se habían esbozado unas líneas gruesas en torno a la formación de actitud científica que respondiera a los tiempos actuales; en ese entonces se decía que: "un estudiante de filosofía, o de ciencia, o de cualquier saber, que asuma la crítica como uno de sus métodos para hallar la verdad, sólo encontrará resultados satisfactorios cuando sea capaz de enunciar sus problemas lo más claramente que pueda y presente la solución en una forma suficientemente definida, o sea, que pueda discutirse racionalmente". Según este planteamiento, se asume que la crítica es fundamental no sólo para la ciencia sino para cualquier saber; de igual forma, se asume que la crítica nos acerca a la verdad y, finalmente, que la verdad hallada en su investigación empírica tiene que estar sometida a la criba de la crítica, más que al aplauso de los pares o de la institución que lo patrocina.

(25) En este punto seguimos al pie de la letra la propuesta de Haack (2007) denominada: Sensismo Común Crítico (Critical Common-Sensism). 
(26) Una relación causal se denomina lineal cuando una serie de proposiciones no regresan, cerrando un círculo, a su punto de inicio; esto implica que nunca el resultado de algo va a ejercer sus efectos sobre su propio origen. Por lo tanto, no intervienen procesos de retroalimentación y la secuencia de las causas y los efectos no retornan al punto de partida (Ceberio y Watzlawick 1998:40).

\section{Bibliografía}

Aguirre, J. C. y Jaramillo, L. G. 2009. La ciencia y el sentido común crítico. Documento de trabajo surgido al interior del grupo Fenomenología y Ciencia. Popayán: Universidad del Cauca.

Aguirre, J. C. 2007. ¿Tiene algún sentido hablar de ciencia? En: De Lara, F. y otros. Sobre ciencia, técnica y sociedad. Popayán: Universidad del Cauca, pp. 1-11.

Bloor, D. 1998. Conocimiento e imaginario social. Barcelona: Gedisa.

Ceberio, M. y Watzlawick, P. 1998. La construcción del universo. Barcelona: Herder

Giere, R. 2005. Scientific realism: Old and new problems. Erkenntnis 63: 149-165.

Giere, R. 1994. Viewing science. En: PSA: Proceedings of the Biennial Meeting of the Philosophy of Science Association, Vol. 1994, Volume Two: Symposia and Invited Papers, pp. 3-16.

Gross, P. y Levitt, N. 1994. Higher superstition. The academic left and its quarrels with science. Baltimore: John Hopkins.

Haack, S. 2007. Defending science -within reason: Between scientism and cynicism. New York: Prometheus Books.

Hacking, I. 1988a. Philosophers of experiment. En: PSA: Proceedings of the Biennial Meeting of the Philosophy of Science Association, Vol. 1988, Volume Two: Symposia and Invited Papers, pp. 147-156.

Hacking, I. 1988b. The participant irrealist at large in the laboratory. The British Journal for the Philosophy of Science 39(3): 277-294.

Hacking, I. 1983. Representing and intervening: Introductory topics in the philosophy of natural science. Cambridge: Cambridge University Press.

Jaramillo, L. G. 2006. Investigación y subjetividad. La complementariedad como posibilidad para investigar en educación. Tesis de Doctorado. Universidad de Tras-os-Montes. Vila Real, Portugal.

Jaramillo, L. G. y Aguirre, J. C. 2004. La controversia Kuhn-Popper en torno al progreso científico y sus posibles aportes a la enseñanza de la ciencia. Cinta moebio 20: 1-20.

Jardine, N. y Frasca-Spada, M. 1997. Splendours and miseries of the science wars. Studies in History and Philosophy of Science 28(2): 219-235.

Jurdant, B. 2003. Imposturas científicas; los malentendidos del caso Sokal. Madrid: Cátedra. 
Kitcher, P. 2001. El avance de la ciencia: ciencia sin leyenda, objetividad sin ilusiones. México: Universidad Nacional Autónoma de México.

Kitcher, P. 1998. A plea for science studies. En: Koertge, N. (ed.) House built on sand: Exposing postmodernist myths about science. Oxford: Oxford University Press, pp. 32-50.

Knor-Cetina, K. 2005. La fabricación del conocimiento: un ensayo sobre el carácter constructivista y contextual de la ciencia. Quilmes: Universidad Nacional de Quilmes.

Koertge, N. 2007. The science wars. Minerva 45: 105-111.

Latour, B. y Woolgar, S. 1979. Laboratory life. The social construction of scientific facts. London: Sage Publications.

Lyotard, J. F. 1989. La condición postmoderna. Madrid: Cátedra.

Maffesoli, M. 1997. Elogio de la razón sensible. Barcelona: Paidós.

Maffesoli, M. 1993. El conocimiento ordinario; compendio de sociología. México: Fondo de Cultura Económica.

Popper, K. 1992. Conocimiento objetivo: un enfoque evolucionista. Barcelona: Paidós.

Searle, J. 1993. Is there a crisis in American higher education? Bulletin of the American Academy of Arts and Sciences 46(4): 24-47.

Sokal, A. 2007. What is science and why should we care? Third Annual Sense About Science lecture. Febrero 27, UCL, Cruciform Lecture Theatre.

Sokal, A. 1998. What the social text affaire does and does not prove. En: Koertge, N. (ed.) House built on sand: Exposing postmodernist myths about science. Oxford: Oxford University Press, pp. 9-22.

Sokal, A. y Bricmont, J. 1999. Imposturas intelectuales. Barcelona: Paidós.

Theocharis, T. 1997. When did the science wars start? Science and Engineering Ethics 3: 271-272.

Van Fraassen, B. 1980. The scientific image. Oxford: Oxford University Press.

Van Fraassen, B. 1977. The argument concerning scientific realism. Los Angeles: University of Southern California.

Recibido el 26 Mar 2010

Aceptado el 15 Junio 2010 